\title{
PRESENÇA DE ORTOREXIA NERVOSA EM ESTUDANTES DE EDUCAÇÃO FÍSICA E NUTRIÇÃO
}

\author{
Mayara Rodrigues da Silva, Paula Lima Fernandes \\ Universidade do Oeste Paulista - UNOESTE, Presidente Prudente, SP. e-mail: mayararms26@gmail.com
}

\section{RESUMO}

O objetivo do estudo foi avaliar a presença de ortorexia nervosa em estudantes de graduação de nutrição e educação física e sua relação com o uso de redes sociais. Trata-se de um estudo transversal, o qual incluiu graduandos de nutrição e educação física de uma universidade do interior de São Paulo, de ambos os sexos, maiores de 18 anos, que concordaram em participar do estudo. Foi utilizado o questionário Orto-15 para avaliação de comportamentos característicos de ortorexia nervosa. Realizou-se também a associação do uso de redes sociais com ortorexia nervosa. Os resultados obtidos mostraram alta prevalência de comportamento ortoréxico $(85,1 \%)$. A prevalência foi maior no curso de educação física $(53,3 \%)$, que tem maioria de alunos do sexo masculino. No curso de nutrição, a prevalência foi de $46,7 \%$. Não houve associação da ortorexia com uso de redes sociais.

Palavras-chaves: Transtornos da alimentação e da ingestão de alimentos, comportamento alimentar, estudantes de ciências da saúde, redes sociais.

\section{PRESENCE OF ORTHOREXIA NERVOSA IN PHYSICAL EDUCATION AND NUTRITION UNDERGRADUATION STUDENTS}

\begin{abstract}
The objective of the study was to evaluate the presence of orthorexia nervosa in undergraduate students of nutrition and physical education and its relation with the use of social networks. This is a cross-sectional study, which included undergraduate students of nutrition and physical education from an university of the São Paulo countryside, both men and women, over 18 years of age, who agreed to attend on the study. The Orto-15 questionnaire was used to evaluate ortorexia nervosa behaviors. The association of social networks use with ortorexia nervosa was also evaluated. The results showed high prevalence of orthorexic behavior (85.1\%). The prevalence was higher in physical education course $(53.3 \%)$, which has majority of male students. Prevalence in nutrition course was $46.7 \%$. There was no association of orthorexia with social networks use.
\end{abstract}

Keywords: Feeding and eating disorders, feeding behavior, students, health occupations, social networking.

\section{INTRODUÇÃO}

Os transtornos alimentares são graves distúrbios psiquiátricos que levam a preocupações exageradas com a alimentação e/ou aparência física. Geralmente, esse tipo de transtorno acomete, em sua maioria, adolescentes e adultos jovens do sexo feminino, porém, não excluindo o sexo masculino, que apresenta uma menor incidência ${ }^{1}$.

Os transtornos alimentares mais popularmente conhecidos são anorexia nervosa e bulimia nervosa. São patologias caracterizadas por alteração de ingestão e absorção alimentar, através da recusa alimentar que leva a perda de 
peso intensa, no caso da anorexia, ou ainda por episódios de uso de método compensatório purgativo, como vômitos induzidos e utilização de laxantes após episódios de compulsão alimentar, no caso da bulimia nervosa ${ }^{1}$.

Segundo a última versão do Manual Diagnóstico e Estatístico de Transtornos Mentais (DSM V), além da anorexia e bulimia, também são considerados transtornos alimentares 0 transtorno de pica, ruminação, compulsão alimentar, e transtorno alimentar restritivo/evitativo ${ }^{2}$.

A ortorexia nervosa não é contemplada como transtorno alimentar segundo esse manual, pois vem sendo reconhecida e divulgada mais recentemente. É caracterizada como uma obsessão por comer saudável, e por isso, visto como uma boa atitude, tanto por quem pratica, quanto pelas pessoas que estão a volta, o que dificulta seu reconhecimento e diagnóstico ${ }^{3}$. Nesse transtorno, a preocupação é focada na qualidade dos alimentos consumidos, e não tanto na quantidade de alimentos ingerida, na perda de massa corporal e imagem corporal como na anorexia nervosa e bulimia nervosa ${ }^{4}$.

O termo ortorexia nervosa é derivado do grego: orthos significa correto e orexis, apetite. ${ }^{5}$ Trata-se de um comportamento patológico em que há obsessão por comer saudável, em que são excluídos da dieta, alimentos considerados não saudáveis, como por exemplo: açúcares, sal, gorduras, alimentos industrializados, corantes, conservantes, pesticidas e derivados de organismos geneticamente modificados. Nessa doença, os ortoréxicos consomem somente alimentos que mantenham o bom funcionamento e que "libertem o corpo e a mente de impurezas" ${ }^{\prime 5,6}$.

Eles dedicam muito tempo diariamente com o planejamento, aquisição, preparação e consumo de suas refeições ${ }^{6}$. Geralmente são pessoas perfeccionistas e ansiosas, e não permitem nenhum tipo de evasão da dieta, sempre mantendo uma rígida restrição $e$ autocontrole. $O$ fato de praticarem uma dieta restritiva pode levar esses indivíduos ao desenvolvimento de carências nutricionais, como anemia, hipovitaminoses, além de osteoporose e desnutrição. As restrições iniciam-se com o objetivo de melhorar a saúde, tratar uma enfermidade ou perder massa corporal, mas a dieta passa a ocupar lugar central na vida. Além disso, esse comportamento também está relacionado ao afastamento social e desprezo com outros indivíduos que não exercem o mesmo estilo de vida ${ }^{6,7}$.

Tanto ortorexia, quanto os transtornos alimentares estão cada vez mais frequentes na sociedade ${ }^{8}$. Os possíveis motivos que levam as pessoas a desencadearem a ortorexia, seriam: a obsessão ao acreditar que seu corpo estará impuro ao comer algo que eles não conhecem, como também procurar muitas informações nutricionais em fontes não confiáveis, pesquisar dietas da moda para emagrecimento, comodismo alimentar e a excessiva publicidade de produtos supostamente saudáveis impostos pela mídia ${ }^{9}$.

A mídia pode fazer com que seu público entenda de forma errada ou influencie de forma negativa as pessoas a escolherem alimentos inapropriados. Ao mesmo tempo, impõe padrões corporais vinculados à saúde alimentar, fazendo com que pessoas fora desses padrões se frustrem e consequentemente desenvolvam transtornos alimentares ${ }^{10,11}$.

O uso de mídias sociais vem crescendo e pode ter um efeito importante na saúde mental $^{12}$. Redes sociais, como por exemplo o Instagram, que é uma plataforma baseada no compartilhamento de fotos e vídeos, tem sido associadas a problemas de saúde mental em adultos jovens, como depressão e distúrbios alimentares ${ }^{12,13}$.

Perfis que abordam conteúdos de saúde, alimentação e o mundo fitness, têm sido amplamente divulgados e recebem reforço positivo dos usuários. Os usuários sofrem influência dessas imagens de famosos, que podem influencia-los negativamente, além de usarem conhecimentos populares em suas descrições, e não evidências científicas ${ }^{14}$.

Além da influência das redes sociais, outro fator de risco para o desenvolvimento de transtornos alimentares ou comportamentos associados é o grupo de estudantes de nutrição e educação física.

Souza et al verificaram em um estudo sobre desenvolvimento de ortorexia nervosa em estudantes de nutrição que houve prevalência de $75 \%$ de presença de tal patologia ${ }^{6}$. Em um estudo realizado com estudantes universitários de educação física, Vital et al. $^{15}$ encontraram comportamento ortoréxico em $82,5 \%$ dos quarenta estudantes avaliados.

Estudantes de nutrição, profissionais da área da saúde, educadores físicos e esportistas são grupos propensos a desenvolverem ortorexia. 
Isso se dá, na maioria das vezes devido à alta cobrança de seguir rígidos hábitos alimentares pelo fato, de se sentirem no dever de passar essa imagem para o público ${ }^{15}$. A presença de distúrbios de imagem corporal nos universitários pode resultar em más influencias em suas futuras práticas profissionais. Além disso, o desenvolvimento de ortorexia nervosa está diretamente relacionado com o desenvolvimento de outros transtornos alimentares ${ }^{16}$.

Por se tratar de uma patologia recémdescoberta e pouco abordada, faltam estudos específicos para diagnóstico desta condição, principalmente em grupos de risco. Portanto, o objetivo deste trabalho foi identificar a prevalência de ortorexia nervosa em estudantes da área de nutrição e educação física, os quais possuem maior susceptibilidade de ocorrência da doença, já que esses estudantes e futuros profissionais possuem uma cobrança exagerada em relação à aparência física.

\section{METODOLOGIA}

Trata-se de um estudo transversal, em que foram incluídos estudantes de graduação em nutrição e educação física de uma universidade do interior de São Paulo, Brasil.

\section{Procedimentos éticos}

Este projeto foi aprovado pelo Comitê de Ética em Pesquisa da Universidade do Oeste Paulista - UNOESTE (CAAE: 87307818.6.0000.5515). Todos os participantes assinaram o Termo de Consentimento Livre e Esclarecido.

\section{Critérios de inclusão}

Foram incluídos nessa pesquisa os estudantes universitários de educação física e nutrição do 1 을 ao 3o ano de graduação, de ambos os sexos, maiores de 18 anos.

Coleta de dados

Os universitários preencheram os questionários avaliativos em sala de aula. O questionário foi respondido de forma anônima, e continha informações como idade, sexo, o uso de redes sociais, o termo frequentado no curso, bem como peso e altura referidos, para posterior cálculo do índice de massa corporal (IMC).

Avaliação de comportamentos característicos de ortorexia
Foi utilizado o questionário Orto-15, composto por 15 perguntas, as quais foram elaboradas por Donini et al. ${ }^{14}$, adaptado por Pontes e Montagner ${ }^{17}$ para a língua portuguesa. Esse questionário teve como objetivo identificar aspectos comportamentais que mostressem a prevalência de comportamentos ortoréxicos, baseados em perguntas simples com respostas objetivas e claras para diagnosticar o público com risco de ortorexia ${ }^{17}$.

Análise estatística

Dados foram expressos em média e desvio padrão. Frequências foram expressas em porcentagens. A comparação entre os grupos com e sem ortorexia e entre os cursos de nutrição e educação física foi realizada por teste $t$ de Student ou qui-quadrado. Correlação de Spearman foi utilizada para avaliar associação da pontuação no questionário Orto-15 com idade e IMC. Significância estatística foi considerada quando $p<0,05$.

\section{RESULTADOS}

Foram incluídos 195 indivíduos, cujas características gerais estão listadas na Tabela 1. A idade variou de 18 a 54 anos.

Tabela 1. Características gerais dos indivíduos incluídos ( $n=195)$.

\begin{tabular}{lc}
\hline Característica & $\mathbf{N}(\%)$ \\
\hline Sexo & \\
Masculino & $86(44,3)$ \\
$\begin{array}{l}\text { Feminino } \\
\text { Curso }\end{array}$ & $108(55,7)$ \\
Educação física & $96(49,5)$ \\
Nutrição & $98(50,5)$ \\
Ano de Graduação & \\
1o & $46(23,6)$ \\
2o & $72(37,2)$ \\
3o & $76(39,1)$ \\
& \\
Idade $(\mathrm{anos})$ & $22,6 \pm 5,0$ \\
IMC (Kg/m²) & $24,1 \pm 9,0$ \\
\hline
\end{tabular}

Considerando o ponto de corte de 40 pontos no questionário Orto-15, foi encontrada a prevalência de $85,1 \%$ de ortorexia (166 alunos). A partir desse ponto de corte, os alunos foram divididos em dois grupos (com e sem ortorexia) e comparados (Tabela 2). Um dos participantes não respondeu às perguntas relacionadas às características, por isso a descrição das 
características na Tabela 2, o grupo com ortorexia inclui 165 participantes. Apenas 4 indivíduos $(2,1 \%)$ não utilizavam nenhum tipo de rede social.

Foi realizada também a comparação entre os participantes de acordo com o curso de graduação. Foi encontrada diferença em relação ao sexo, prevalência de ortorexia e ano da graduação.

Foi encontrada correlação negativa e significativa entre a pontuação obtida no questionário Orto-15 com IMC, ou seja, quanto menor o IMC, maior a pontuação no questionário.

Tabela 2. Comparação entre os grupos com e sem ortorexia.

\begin{tabular}{lccc}
\hline & $\begin{array}{c}\text { Com ortorexia } \\
(\mathrm{n}=165) \mathrm{N}(\%)\end{array}$ & $\begin{array}{c}\text { Sem ortorexia } \\
(\mathrm{n}=29) \mathrm{N}(\%)\end{array}$ & $\mathbf{P}$ \\
\hline Sexo & $78(47,3)$ & $8(27,6)$ & 0,49 \\
$\begin{array}{l}\text { Masculino } \\
\text { Feminino }\end{array}$ & $87(52,7)$ & $21(72,4)$ & \\
$\begin{array}{l}\text { Curso } \\
\text { Educação física }\end{array}$ & $88(53,3)$ & $8(27,6)$ & 0,11 \\
Nutrição & $77(46,7)$ & $21(72,4)$ & \\
$\begin{array}{l}\text { Ano da Graduação } \\
\text { 1o }\end{array}$ & $41(24,8)$ & $5(17,2)$ & \\
2o & $65(39,4)$ & $7(24,1)$ & 0,066 \\
3o & $59(35,8)$ & $17(58,6)$ & \\
& & & \\
Uso do Instagram & $132(80,0)$ & $22(75,9)$ & 0,611 \\
& & & \\
Idade & $22,6 \pm 4,8$ & $22,5 \pm 6,0$ & 0,92 \\
IMC (kg/m ( $^{2}$ ) & $24,3 \pm 9,6$ & $22,8 \pm 4,3$ & 0,40 \\
Pontuação Orto-15 & $34,51 \pm 3,53$ & $42 \pm 1,98$ & 0,0001 \\
\hline
\end{tabular}

Tabela 3. Comparação entre os cursos educação física e nutrição

\begin{tabular}{lccc}
\hline & $\begin{array}{c}\text { Educação física } \\
(\mathrm{n}=96) \mathrm{N}(\%)\end{array}$ & $\begin{array}{c}\text { Nutrição } \\
(\mathrm{n}=98) \mathrm{N}(\%)\end{array}$ & $\mathbf{P}$ \\
\hline Sexo & $69(71,9)$ & $17(17,3)$ & 0,0001 \\
$\begin{array}{l}\text { Masculino } \\
\text { Feminino }\end{array}$ & $27(28,1)$ & $81(82,7)$ & \\
Curso & & & \\
Educação física & $88(53,3)$ & $8(27,6)$ & 0,11 \\
Nutrição & $77(46,7)$ & $21(72,4)$ & \\
Ano da Graduação & & & \\
10 & $29(30,2)$ & $17(17,3)$ & \\
20 & $52(54,2)$ & $20(20,4)$ & 0,0001 \\
3o & $15(15,6)$ & $61(62,2)$ & \\
& & & \\
Ortorexia & $88(91,7)$ & $77(78,6)$ & 0,011 \\
Uso do Instagram & $71(74,0)$ & $83(84,7)$ & 0,065 \\
& & & \\
Idade & $22,6 \pm 4,8$ & $22,5 \pm 5,04$ & 0,965 \\
IMC (kg/m ${ }^{2}$ ) & $25,3 \pm 12,12$ & $22,77 \pm 3,8$ & 0,44 \\
Pontuação Orto-15 & $35,19 \pm 3,9$ & $36,11 \pm 4,5$ & 0,132 \\
\hline
\end{tabular}



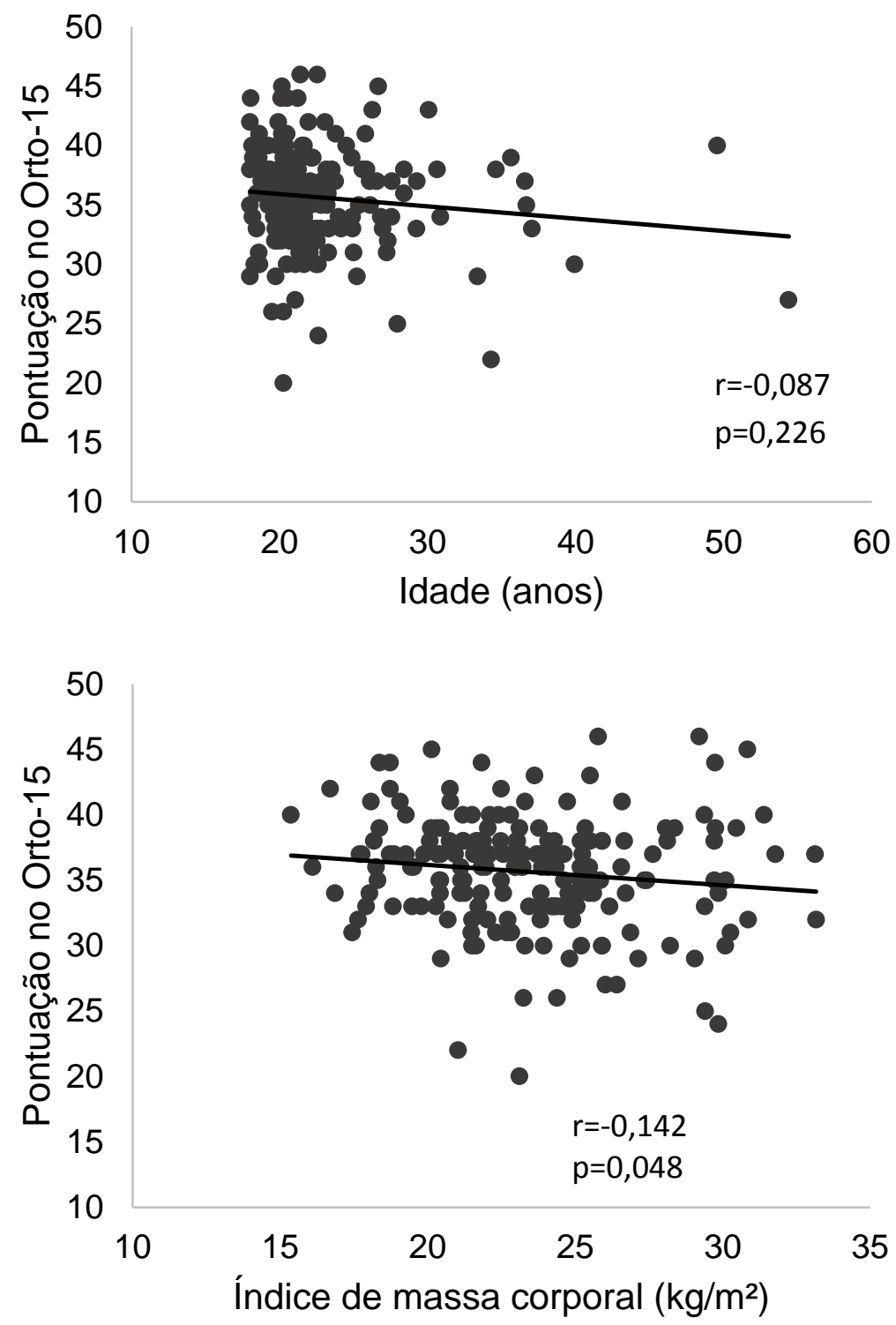

Figura 1. correlações entre a pontuação obtida no questionário Orto-15 com idade (superior) e índice de massa corporal (inferior; $n=195$ ).

\section{DISCUSSÃO}

Foi identificada alta prevalência nesse grupo de universitários estudantes de Educação Física e de Nutrição. Contudo, não houve significância na relação com o uso de redes sociais.

Houve alta prevalência de ortorexia nervosa no presente estudo, visto que os profissionais da área da saúde então mais comprometidos com a adoção de hábitos de vida mais saudáveis, incluindo hábitos alimentares. Consequentemente, estão pré-dispostos a desenvolverem ortorexia com maior facilidade, comparados a outros profissionais ${ }^{18}$.

Bressan et al. ${ }^{19}$, em uma revisão bibliográfica que incluiu quinze artigos, observaram que há influência da mídia nos indivíduos da sociedade contemporânea, como um fator promotor de transtornos devido a conflitos causados entre sua imagem real e imagem idealizada.

No presente estudo foi verificado que os indivíduos dos termos iniciais apresentaram maior presença de comportamentos ortoréxicos do que nos termos finais. A hipótese levantada é que no início da graduação, os graduandos apresentam maior disposição comparado com os que estão prestes a se formar. Levando em conta a aparência física, como uma propaganda da sua futura profissão ou simplesmente de serem "influencers" do curso, dedicando mais do seu tempo à alimentação e a prática de atividades físicas. Entretanto, mais ao fim da graduação devido à falta de tempo e afazeres dos cursos em 
questão, o cuidado com a aparência e preocupação alimentar já não é o mesmo. Não é do nosso conhecimento trabalhos que tenham verificado a influência do período da graduação no desenvolvimento de ortorexia.

Nosso estudo mostrou que há maior prevalência de ortorexia no curso de educação física, o qual possui maior número de pessoas do sexo masculino. Rocha et al. ${ }^{21}$, mostraram que o grupo considerado de risco para desenvolvimento de ortorexia são estudantes da área da saúde, e alguns grupos de atletas, como fisiculturistas e praticantes de atletismo. Quando os questionários foram aplicados, foi observado que parte dos graduandos de educação física levantaram perguntas pertinentes a estarem em fase de competições. Nessa pesquisa, não foi avaliado se os indivíduos eram atletas. Porém, no curso de educação física, uma parcela significante dos alunos se interessa por atividade física e competições, podendo isto, representar um grupo de risco.

Além disso, na grade curricular de Educação Física, os alunos possuem apenas uma matéria relacionada à nutrição, especifica para a área de nutrição esportiva. Ou seja, o conhecimento em nutrição poderia levar a menor prevalência de ortorexia nervosa, já que quando comparados ao curso de nutrição, a educação física apresentou maiores índices de ortorexia.

Em nosso estudo, não houve diferença em relação aos sexos entre os indivíduos com e sem ortorexia. Na literatura, é relatada que a insatisfação com a imagem corporal é mais comum entre o sexo feminino ${ }^{19}$, porém ao analisar o curso de nutrição que possui grande maioria do sexo feminino, a prevalência de ortorexia é menor do que na educação física, que por sua vez apresentou como maioria o sexo masculino.

A vigorexia também ainda não considerada um transtorno alimentar, é caracterizada pela pratica exaustiva de exercícios físicos, com objetivo de busca por um corpo perfeito $^{22}$. Afeta principalmente homens jovens ${ }^{22}$, características semelhantes aos graduandos do curso de educação física. Na vigorexia, a busca pelo corpo ideal o indivíduo se submete a dietas restritas. Por isso, as preguntas e respostas do questionário Orto-15 podem não diferenciar essas duas condições.

Esse estudo possui limitações como, o cálculo do IMC, que se faz através de valores de peso e altura relatados, podendo ocasionar divergências se comparados a outros estudos.

Em conclusão, foi encontrada alta prevalência de ortorexia nervosa em estudantes de graduação em nutrição e educação física, relacionada em sua maioria com o sexo masculino e curso de educação física, entretanto, não foram encontradas associações entre o uso de redes sociais e o comportamento ortoréxico.

\section{CONFLITO DE INTERESSE}

Os autores declaram não haver qualquer potencial conflito de interesse que possa interferir na imparcialidade deste trabalho científico.

\section{REFERÊNCIAS}

1. Cordás TA, Rios SR, Salzano FT. Os transtornos alimentares e a evolução no diagnóstico e no tratamento. Alvarenga $\mathrm{M}$, Philippi ST. Transtornos alimentares: uma visão nutricional. Barueri: Manole; 2004.

2. Cordioli AV. Manual diagnostico e estatístico de transtornos mentais: DSM-5 /American Psychiatric Association. Porto Alegre: Artmed; 2014.

3. Matos RAC, Nascimento PAPM, Rodrigues RA. Características comportamentais de ortorexia nervosa em estudantes do curso de nutrição. Nutr Pauta. 2013;13:1-5.

4. Lopes MR, Kirsten VR. Comportamentos de ortorexia nervosa em mulheres jovens. Disciplin Scien. 2009;10(1):97-105.

5. Bratman SMD. Original essay on orthorexia, 1997. Disponível em: http://www.orthorexia.com

6. Souza QJOV, Alexandra AM. Comportamento de risco para ortorexia nervosa em estudantes de nutrição. J Bras Psiquiatr. 2014;63(3):200-4. DOI: https://doi.org/10.1590/0047-2085000000026

7. Martins MCT, Alvarenga MS, Vargas SVA, Sato KSCJ, Scagliusi FB. Ortorexia nervosa: reflexões sobre um novo conceito. Rev Nutr. 2011;24(2):345-57. DOI: https://doi.org/10.1590/S1415$\underline{52732011000200015}$

8. Coelho GC, Trogilo GM, Hammes L, Galvão TD, Cyrino LAR. As consequências físicas, psíquicas e 
sociais em indivíduos com ortorexia nervosa. Rev Bras Obes Nutr Emagrec. 2016;57(10):160-6.

9. Castro AL. Culto ao corpo e estilos de vida: o jogo da construção de identidades na cultura contemporânea. Perspectivas. 2007;31(1):13768.

10. Teixeira MMC, Santos AM, Alves VSV, Jesus SKSC, Baeza SF. Ortorexia nervosa: reflexões sobre um novo conceito. Rev Nutr. 2017;24(2):345-57. DOI: https://doi.org/10.1590/S1415$\underline{52732011000200015}$

11. Carrotte ER, Vella AM, Lim MS. Predictors of liking three types of health and fitness-related content on social media: a cross-sectional study. J Med Res. 2015;17(8):e205. DOI: https://doi.org/10.2196/imir.4803

12. Sidani JE, Shensa A, Radovic A, Miller $E$, Colditz JB, Hoffman BL, Giles LM, Primack BA. Association between social media use and depression among US young adults. Depress Anxiety. 2016;33(4):323-33. DOI: https://doi.org/10.1002/da.22466

13. Donini L, Marsili D, Graziani M, Imbriale M, Cannella C. Orthorexia nervosa: validation of a diagnosis questionnaire. Eat Weight Disord. 2005;10(2):28-32.

DOI: https://doi.org/10.1007/BF03327537

14. Tiggemann M, Zaccardo M. 'Strong is the new skinny': a content analysis of fitspiration images on Instagram. J Health Psychol. 2018;23(8):100311. DOI: https://doi.org/10.1177/1359105316639436

15. Vital ANS, Silva ABA, Silva EIG. Risco para desenvolvimento para ortorexia nervosa e o comportamento alimentar em estudantes universitário. Saúde Pesq. 2017;10(1):83-9. DOI: https://doi.org/10.17765/1983$\underline{1870.2017 v 10 n 1 p 83-89}$

16. Luna CA, Belmonte TSA. Ortorexia Nervosa: um desafio para o nutrólogo. Int J Nutrol. 2016;9(1):128-39.

17. Montagner MI, Montagner MA, Pontes JB. Ortorexia nervosa: adaptação cultural do Orto-
15. Demetra. Planaltina. 2014;9(2):533-48. DOI: https://doi.org/10.12957/demetra.2014.8576

18. Souza, JMP. Ortorexia nervosa em nutricionistas e estudantes de nutrição. [Monografia]. Pós-Graduação em Nutrição, Universidade de Brasília; 2017.

19. Bressan MR, Pujals C. Transtornos alimentares modernos: uma comparação entre ortorexia e vigorexia. Rev Uningá Rev. 2015;23(3):25-30.

20. Alvarenga MS, Martins MCT, Sato KSCJ, Vargas SVA, Philippi ST, Scagliusi FB. Orthorexia nervosa behavior in a sample of brazilian dietitians assessed by the portuguese version of ORTO-15. Eating Weight Disord Studies Anorexia, Bulimia Obes. 2012;17(1):29-35. DOI: https://doi.org/10.1007/BF03325325

21. Rocha MAP, Barthichoto M, Lopes JE. Costa K, Viebig RF. Ortorexia: uma compulsão por alimentos saudáveis. Nutr Brasil. 2016;1(14).

22. Soler PT, Fernandes HM, Damasceno VO, Novaes JS. Vigorexia e níveis de dependência de exercício em frequentadores de academias e fisiculturistas. Rev Bras Med Esporte. 2013;19(5):343-8. DOI: https://doi.org/10.1590/S1517$\underline{86922013000500009}$ 